\title{
Estimation of an inter-rater intra-class correlation coefficient that overcomes common assumption violations in the assessment of health measurement scales
}

\author{
Carly A. Bobak ${ }^{1+}$ (D), Paul J. Barr ${ }^{2}$ and A. James O'Malley ${ }^{1,2^{*}+}$
}

\begin{abstract}
Background: Intraclass correlation coefficients (ICC) are recommended for the assessment of the reliability of measurement scales. However, the ICC is subject to a variety of statistical assumptions such as normality and stable variance, which are rarely considered in health applications.

Methods: A Bayesian approach using hierarchical regression and variance-function modeling is proposed to estimate the ICC with emphasis on accounting for heterogeneous variances across a measurement scale. As an application, we review the implementation of using an ICC to evaluate the reliability of Observer OPTION ${ }^{5}$, an instrument which used trained raters to evaluate the level of Shared Decision Making between clinicians and patients. The study used two raters to evaluate recordings of 311 clinical encounters across three studies to evaluate the impact of using a Personal Decision Aid over usual care. We particularly focus on deriving an estimate for the ICC when multiple studies are being considered as part of the data.

Results: The results demonstrate that ICC varies substantially across studies and patient-physician encounters within studies. Using the new framework we developed, the study-specific ICCs were estimated to be $0.821,0.295$, and 0.644. If the within- and between-encounter variances were assumed to be the same across studies, the estimated within-study ICC was 0.609 . If heteroscedasticity is not properly adjusted for, the within-study ICC estimate was inflated to be as high as 0.640 . Finally, if the data were pooled across studies without accounting for the variability between studies then ICC estimates were further inflated by approximately 0.02 while formerly allowing for between study variation in the ICC inflated its estimated value by approximately 0.066 to 0.072 depending on the model.

Conclusion: We demonstrated that misuse of the ICC statistics under common assumption violations leads to misleading and likely inflated estimates of interrater reliability. A statistical analysis that overcomes these violations by expanding the standard statistical model to account for them leads to estimates that are a better reflection of a measurement scale's reliability while maintaining ease of interpretation. Bayesian methods are particularly well suited to estimating the expanded statistical model.
\end{abstract}

Keywords: Bayesian analysis, Hierarchical regression, Variance function modelling, Reliability, ICC, Shared decision making, Observer OPTION 5

*Correspondence: James.OMalley@dartmouth.edu

${ }^{\dagger}$ Carly A. Bobak and A. James O'Malley contributed equally to this work.

${ }^{1}$ Department of Quantitative Biomedical Sciences, Geisel School of Medicine,

Dartmouth College, 1 Rope Ferry Road, 03755 Hanover, NH, USA

${ }^{2}$ The Dartmouth Institute, Geisel School of Medicine, Dartmouth College,

1 Rope Ferry Road, 03755 Hanover, NH, USA

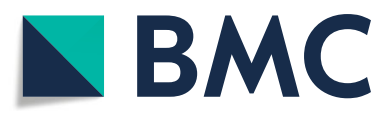

(c) The Author(s). 2018 Open Access This article is distributed under the terms of the Creative Commons Attribution 4.0 International License (http://creativecommons.org/licenses/by/4.0/), which permits unrestricted use, distribution, and reproduction in any medium, provided you give appropriate credit to the original author(s) and the source, provide a link to the Creative Commons license, and indicate if changes were made. The Creative Commons Public Domain Dedication waiver (http://creativecommons.org/publicdomain/zero/1.0/) applies to the data made available in this article, unless otherwise stated. 


\section{Background}

R. A. Fisher first introduced the concept of an intraclass correlation coefficient (ICC) in his 1921 paper examining the familial resemblance between siblings [1]. Since then, it has become an important measurement used in the fields of psychology, genetic linkage, heritability, sensitivity analysis, study design, DNA micro array analysis, and health measurement scales [2-11]. The ICC is a measure of reliability, specifically the reliability of two different raters to measure subjects similarly [12, 13]. Inter-rater reliability is important as it demonstrates that a scale is robust to changes in raters. Hence, scales with high inter-rater reliability are less prone to measurement error such as caused by variation in human judgement [13].

In the area of health measurement scales, the ICC has been integrated into the Consensus-based Standards for the selection of the health status measurement instruments (COSMIN) check list. This checklist was developed to assess the methodological quality of studies based on measurement attributes. One of the major boxes on the COSMIN check list is reliability, where it is recommended that the ICC be used as a measurement of inter-rater reliability $[9,10]$. One of the driving factors of the uptake of the ICC in many fields is its ease of interpretation $[9,10]$. The ICC is a value between 0 and 1 , where values below 0.5 indicate poor reliability, between 0.5 and 0.75 moderate reliability, between 0.75 and 0.9 good reliability, and any value above 0.9 indicates excellent reliability [14].

However, it has been established that the ICC is subject to a variety of methodological issues [1, 15-22]. These include a sensitivity to assumptions regarding normality and homogeneous variance, as well as having a negatively biased maximum likelihood estimator and least squares estimator [1, 18, 20-22]. A variety of methods have been proposed to address these concerns. Various variance-stabilizing transformations have been proposed [1, 19], as well as bias-corrected estimators [22, 23]. There are few factors which limit the uptake of such methods. First, guidance of how to properly implement the ICC is not communicated clearly, particularly to non-statisticians. Secondly, ease of interpretation is of utmost importance with the ICC measure, and while transformations either pre-analysis or internal to a model can correct for heterogeneity in the variance of the measurement across its true value or across other variables, they do so at the cost of interpretability. This has led to problematic misuses of ICCs in a variety of disciplines, including the evaluation of health measurement scales. Herein we formulate solutions to these problems using the evaluation of an instrument for measuring shared decision making (SDM) for illustration.

\section{Case study background: health measurement scales and observer OPTION ${ }^{5}$}

Employing shared decision making between clinicians and patients has been linked to improvements in patient satisfaction, reduced decisional regret, and emerging evidence of improved treatment engagement [24]. Shared decision making (SDM) can be defined as a process by which patients and clinicians make decisions together, accounting for patient preferences and values in light of the best available evidence for treatment options [25].

Measuring the quality of shared decision making implementations in clinical settings is a challenging task [26]. Patient reported measures are common, but vary in length and quality of psychometric properties. They may also be prone to biases, such as "halo" effects leading to ceiling effects in measurement [27]. Observer measures, where trained raters evaluate shared decision making may be more accurate [28].

The Observer OPTION ${ }^{5}$ tool is a proposed improvement of the Observer OPTION ${ }^{12}$ tool $[29,30]$. The Observer OPTION ${ }^{12}$ tool has been well-established for measuring shared decision making practices but has had mixed inter-rater reliability. It has been criticized for not placing enough emphasis on the patient's role in the SDM process $[29,30]$. The Observer OPTION $^{5}$ instrument aims to ameliorate these shortcomings.

The Observer OPTION ${ }^{5}$ tool is tightly focused on the idea of a collaborative deliberation model [29-31]. It's a five item tool which produces a score between 0 and 20 , which can be rescaled to 0 and 100 for interpretability. The patients rate the clinicians interactions in each of the five areas, giving a score between 0 and 4 , where 0 is no effort and 4 is exemplary effort $[29,30]$. The five items are as follows:

1 The clinician indicates to the patient that there are treatment options,

2 The clinician aids in the informing and deliberating process for the patient,

3 The clinician explores the pros and cons of the treatment options with the patient,

4 The clinician attempts to ascertain the patient's preferences in response to potential treatments discussed,

5 The clinician attempts to integrate the patient's preferences in finalizing the treatment plan.

For a measure of an instrument's reliability to have meaning, there ought to be a standard population against which to assess the accuracy or consistency of the rater scores across encounters. However, in the case of health measurement scales, data is often pooled from multiple studies leading to a "wild west" with no standard population. Indeed, in the SDM application, encounters can 
occur across various institutions and in a variety of settings. We argue that the traditional calculation of the ICC, which relies on the assumption that the variance between encounters is reasonably homogeneous, is too inflexible $[1,15-22]$. As well, the common implementation of a bounded scale, or a scale bounded from below, in health measurement scales often leads to heteroscedasticity. We present a measure of ICC and method for estimating it that generalizes the incumbent approach to account for both heterogeneous data and heteroscedastic variances.

To allow an ICC that caters to multiple contexts to be estimated using a general strategy, we develop a Bayesian model and computational procedure. A desirable feature of Bayesian computations is that they avoid the reliance on analytic approximations when computing estimates. This is particularly pertinent in models that include variance functions or other terms that introduce parameters in nonlinear forms, place constraints on parameters, or estimate nonlinear functions of parameters such as ICCs. It is known that Bayesian estimates can be more precise than their frequentist counterparts, especially when prior information is informative [32]. By illustrating our approach on an evaluation of the increasingly popular Observer OPTION ${ }^{5}$ tool, we hope to catalyze the adoption of more meaningful and informative computations of ICC across all health measurement scale applications.

While we use OPTION ${ }^{5}$ as a running example, the proposed methodology applies to any data collected on a bounded response scale for which the agreement between raters is sought.

The remainder of this paper is organized as follows. We provide a brief background regarding the classical form of the ICC and illustrate how it can be over-estimated when between study variability is large in the "Methods" section. In the "Case study design" section we give an overview of the study conducted to assess the Observer OPTION ${ }^{5}$ tool and reveal the areas of statistical misuse that compromise the measures of ICC that have been previously reported. In the "Bayesian framework" section we propose the Bayesian model that estimates an ICC which incorporates data from heterogeneous populations and allows the variance of the measurements to be heteroscedastic. The "Evaluation of Bayesian Estimation" section details our process for computing our estimates, as well as three different scenarios we used to compare the estimates of our ICC. Our results from the original study, as well as our three scenarios are presented in the "Results" section. A brief discussion of these results and their impact on future research in health measurement scales is included in the "Discussion" section.

\section{Methods}

The ICC is mathematically defined as:

$$
I C C=\frac{\sigma_{b}^{2}}{\sigma_{b}^{2}+\sigma_{w}^{2}}=\left(1+\frac{\sigma_{w}^{2}}{\sigma_{b}^{2}}\right)^{-1}
$$

where $\sigma_{b}^{2}$ is the variance between encounters, and $\sigma_{w}^{2}$ is the variance of the raters within encounters. Similarly, inter-rater reliability with $k$ raters is denoted as:

$$
R=\frac{\sigma_{b}^{2}}{\sigma_{b}^{2}+\frac{\sigma_{w}^{2}}{k}}=\left(1+\frac{\sigma_{w}^{2}}{k \sigma_{b}^{2}}\right)^{-1}
$$

Notice that (1) and (2) increase as $\sigma_{b}^{2}$, the variance between encounters, increases. This can be a potential flaw in the calculation of the ICC, as the measure is artificially inflated when the variance between different patient encounters is larger than will occur in the intended application of the instrument. For example, by pooling data from diverse study subjects, a measure will accurately discriminate between a greater proportion of subjects, inflating the ICC or reliability.

\section{Bayesian framework}

Let $h=1, \ldots, M, i=1, \ldots, N_{h}$, and $j=1, \ldots, R$ be our indices for the study, the patient-physician encounter, and the rater. In the OPTION ${ }^{5}$ analysis the number of studies is $M=3$, the number of encounters within the three studies are $\left(N_{1}=201, N_{2}=72, N_{3}=38\right)$, and the number of raters is $R=2$ although the methodology applies to all values of these. Let $Y$ denote the OPTION ${ }^{5}$ score divided by 100 (for ease of interpretation), $\theta$ the true amount of shared decision making, and $X$ indicate the use of a PDA. Although the effect of $X$ is of interest to this field, our objective is to adjust for it's effect so as to ensure that the evaluations of ICC are meaningful. Our statistical model is

$$
Y_{h i j} \mid \theta_{h i}, X_{h i} \sim \operatorname{Normal}\left(\mu_{h i j}, v_{h i}^{2}\right) I(0,1)
$$

where

$$
I\left\{y_{h i j} \in(0,1)\right\}
$$

restricts the probability distribution of the measured amount of SDM to the interval 0 to 1 , and

$$
\begin{aligned}
\mu_{h i j} & =\theta_{h i}+\beta_{1}(j-1.5)+\beta_{2}\left(X_{h i}-\bar{X}\right) \\
v_{h i}^{2} & =\sigma_{h}^{2} \theta_{h i}\left(1-\theta_{h i}\right)
\end{aligned}
$$

with $\bar{X}$ denoting the sample mean value of $X$. The dependence of $v_{h i}^{2}$ on $\theta_{h i}$ implies that the ICC depends on the true amount of SDM in the encounter; its mathematical expression is referred to as a variance function.

We view the encounters as a random sample from a large population of possible encounters about which we wish to make inferences. The sampling of the encounters and the sampling variability in them is represented mathematically as 


$$
\theta_{h i} \mid \text { study } \sim \operatorname{Normal}\left(\gamma_{h}, \tau_{h}^{2}\right) I(0,1)
$$

where $I$, defined in (4), restricts the possible amount of SDM to be a proportion (0 to 1$)$. The specification for $\theta_{h i}$ depends on parameters which are indexed by $h$, giving each study its own mean and variance. We set our prior distributions as follows:

$$
\begin{aligned}
\gamma_{h} & \sim \operatorname{Normal}\left(\beta_{0}, \omega^{2}\right) \\
\beta_{k} & \sim \operatorname{Normal}\left(b_{0} I(k=0), B^{2}\right), \quad k=0,1,2 \\
\sigma_{h}^{-2} & \sim \operatorname{Gamma}\left(v_{1}, v_{1}\right) \\
\tau_{h}^{-2} & \sim \operatorname{Gamma}\left(v_{2}, v_{2}\right) \\
\omega^{-2} & \sim \operatorname{Gamma}\left(v_{3}, v_{3}\right)
\end{aligned}
$$

The choice of normal and gamma distributions for the regression (mean or location) and the variance (scale) parameters is common in practice as the conditional posterior distributions of each parameter conditional on the remaining parameters and the data are also normal and gamma distributions. This simplifies model estimation and computation.

The desire for the prior distribution to impart virtually no information onto the analysis is accomplished by specifying distributions with very large variances for the model parameters. As a consequence, the data are solely responsible for estimating the model. In this application we set $b_{0}=0.4, B^{2}=10$, and $v_{l}=10^{-3}$ for $l=1,2,3$. Note that parameters such as $\theta_{h i}$ that have restricted ranges may be assigned prior distributions with almost no mass within the allowable range if the density is not truncated. If the allowed range is a region over which the unrestricted distribution is essentially flat, then the truncated distribution will be close to uniform - essentially assuming that all allowable values of the parameter are equally likely. As well, parameters may have values such that the mean of the unrestricted distribution is outside the allowed range, and the truncated distribution will still be well-defined. Although the inverse-Gamma prior distributions assumed here for the variance parameters have been shown to yield undesirable results in some applications [33], we found that they were well suited to our case study in the sense that the results were quite robust to the prior distribution parameters. For example, the results with $v_{l}=10^{-2}$ for $l=1,2,3$ were numerically almost identical to those with $v_{l}=10^{-3}$ for $l=1,2,3$. We attribute this result to the fact that in our case study the scale of the data has a finite range, which prevents the tails of a prior distribution from having a substantial impact on the posterior.

Under the above model, the ICC for an encounter in study $h$ with $\operatorname{SDM}$ of $\theta^{*}$ is given by

$$
\operatorname{ICC}_{h}\left(\theta^{*}\right)=\frac{\tau_{h}^{2}}{\tau_{h}^{2}+\sigma_{h}^{2} \theta^{*}\left(1-\theta^{*}\right)}
$$

Two salient features are evident in Eq. (8). Firstly, the within $\left(\sigma^{2}\right)$ and between $\left(\tau^{2}\right)$ encounter variance and scale parameters depend on the index for study. Therefore, the ICC is study specific. Secondly, the within-encounter scale parameter is multiplied by $\theta^{*}\left(1-\theta^{*}\right)$, which crucially allows for the ability of raters to agree, or rate consistently, to depend on the actual amount of SDM. Because it is easier to distinguish cases against a baseline level of a trait close to $0 \%$ or $100 \%$ than cases in which the trait is about $50 \%$ present (this is seen from the fact that the variability of a restricted scale is greatest around its middle point), the involvement of the binomial variance form $\theta^{*}\left(1-\theta^{*}\right)$ makes intuitive sense.

In practice, one may choose a value of $\theta^{*}$ that has particular meaning or relevance to the application at which to compute the ICC. If multiple values of $\theta^{*}$ are important (e.g., the baseline levels for various population strata) a separate ICC can be reported for each of them. Alternatively, or additionally, one may also choose to average over a population of values of $\theta^{*}$. For example, if we expect the population of patient-physician encounters on which the instrument will be applied to be described by the probability distribution,

$$
\theta^{*} \sim \pi\left(\theta^{*}\right)=\operatorname{Normal}\left(\gamma_{h}, \tau_{h}^{2}\right) I(0,1),
$$

it follows that the population average ICC, given by

$I C C_{h}=\tau_{h}^{2} \int_{0}^{1}\left(\tau_{h}^{2}+\sigma_{h}^{2} \theta^{*}\left(1-\theta^{*}\right)\right)^{-1} \pi\left(\theta^{*}\right) \quad d \theta^{*}$,

should be computed. The evaluation of multiple measures of ICC yields a much more informative profile of an instrument's performance than the presently used single number summary derived under overly restrictive assumptions. This function is designed in such a way that the user directly specifies a distribution for $\theta^{*}$ to maintain flexibility in the calculation of the ICC. This distribution can be specified with known parameters to avoid integration over the hyper parameters $\gamma_{h}$ and $\tau_{h}$ for simplicity. Alternatively, the user could assume a hierarchical prior where integration over these parameters would also be necessary.

The ICC can also be defined for a scenario where encounters are pooled across studies. Assuming an equal probability of selecting an encounter from each study, the marginal variance across these encounters is $\omega^{2}+\bar{\tau}^{2}+$ $\bar{\sigma}^{2} \theta^{*}\left(1-\theta^{*}\right)$ (a more general expression may be substituted if the study selection probabilities are unequal). Hence, the corresponding measure of ICC is given by

$$
I C C_{\mathrm{Marg}}\left(\theta^{*}\right)=\frac{\omega^{2}+\bar{\tau}^{2}}{\omega^{2}+\bar{\tau}^{2}+\bar{\sigma}^{2} \theta^{*}\left(1-\theta^{*}\right)}
$$

Typically, one would see

$$
\operatorname{ICC}_{\mathrm{Marg}}\left(\theta^{*}\right) \geq \overline{\operatorname{ICC}}\left(\theta^{*}\right)
$$


Although the pooled or marginal ICC is well-defined under a specified model for sampling encounters from the individual studies, if the intended use of the instrument is to compare encounters across a homogeneous population of subjects (e.g., the reference population for a single study) then $\operatorname{ICC}_{\text {Marg }}\left(\theta^{*}\right)$ makes the instrument look better in a meaningless way as it overstates the heterogeneity between the subjects compared to the heterogeneity between the individuals in the population that the instrument will be used to compare or discriminate between in actual practice.

Summarizing the above, the three forms of ICC are seen to be components of a two-dimensional family of measures of ICC defined under the full statistical model we developed to account for the intricacies of the data. The dimensions are: 1 ) whether or not the ICC is specific to a particular level of the quantity being studied versus averaging over a distribution of values of that quantity; 2) whether or not variability between studies is included in the between encounter variance (which corresponds to whether or not it is desired for the instrument to discriminate between encounters from different studies in practice). Combining these two dimensions, there are four general types of ICC that are available under the general approach we have proposed.

\section{Evaluation of Bayesian Estimation}

All analyses of the Observer OPTION ${ }^{5}$ data were conducted in R [34]. Markov Chain Monte-Carlo (MCMC) simulation for Bayesian estimation was implemented using Just Another Gibbs Sampler (JAGS) and integrated with pre- and post-processing using the R package 'rjags' $[35,36]$. Three Bayesian models for three separate ICC scenarios are compared. The first is the full model, which separately calculates the posterior variance and ICC for each study. The second restricts the variances to be homogeneous across the three studies. The third ignores the issue of within-study heteroscedasticity in the variability of raters' assessments of the amount of SDM. Posterior distributions are summarized by their median and $95 \%$ symmetric credible interval (2.5th and 97.5th percentiles).

\section{Case study design}

Our main study of interest can be found in [30]. Data was collected from two previous studies, the Chest Pain Choice trial (Study 1) and the Osteroporosis Choice Randomized trial (Studies 2 and 3) [37, 38]. Both trials randomly assigned patients to either receive an intervention of use of a Personal Decision Aid (PDA), or receive usual care [37, 38]. The Osteoporosis Choice Randomized trial contains a subgroup of participants who used the World Health Organization's Fracture Risk Assessment Tool $\left(\right.$ FRAX ${ }^{\oplus}$ ) [38]. For the purposes of our analysis, we consider patients who used FRAX ${ }^{\circledast}$ as a separate study group (Study 3). The Chest Pain Choice trial recruited participants from St. Mary's Hospital Mayo Clinic in Rochester, MN while the Osteoporosis Choice Randomized trial recruited from 10 general care and primary care practices in the Rochester, MN area [37, 38].

Audio-visual recordings of the patient-clinician encounters took place and two-raters independently assessed the recording of each patient-physician encounter across these three clinical studies of decision-aids using the Observer OPTION ${ }^{5}$ SDM tool. A total of 311 clinical encounters were included in the study Table 1 summarizes these encounters across the three studies of interest. The overall Observer OPTION ${ }^{5}$ score was calculated for each encounter and rater [30]. The goal of the following analysis is to determine the concordance of the two raters despite the heterogeneity of the study groups and inherent heteroscedasticity.

In this particular case, the recorded encounters from all three studies were re-rated by the same two raters. Hence, we assume that the differences across the studies are due to the differences in populations and imposed interventions across each study. In many cases, there would also be heterogeneity across raters of studies, leading to even greater between-study heterogeneity than is observed in this case.

\section{Results}

The results from the rater's independent assessment of SDM using Observer OPTION ${ }^{5}$ are shown in Fig. 1. In general, Rater 1 consistently scored encounters higher than Rater 2.

Figure 2 shows the actual difference of Observer OPTION $^{5}$ scores as a function of the mean Observer OPTION $^{5}$ score for each encounter. The mean difference across the encounters is approximately 10 points, although over the range of 30-60 the sample differences were consistently on the order of 12 to 16 and as high as 19.

Figure 3 shows the empirical variance functions. The observed variance connected with a smoothing spline with 10 degrees-of-freedom is shown in the solid line, while the mean and binomial variance functions are shown in dashed lines. It is clearly demonstrated that the

Table 1 Encounters from the three randomized studies which compared the impact of PDAs to standard care

\begin{tabular}{llll}
\hline Study & PDA $(n)$ & Usual care $(n)$ & Total \\
\hline 1 & 101 & 100 & 201 \\
2 & 37 & 35 & 72 \\
3 & 13 & 25 & 38 \\
Total & 151 & 160 & 311 \\
\hline
\end{tabular}




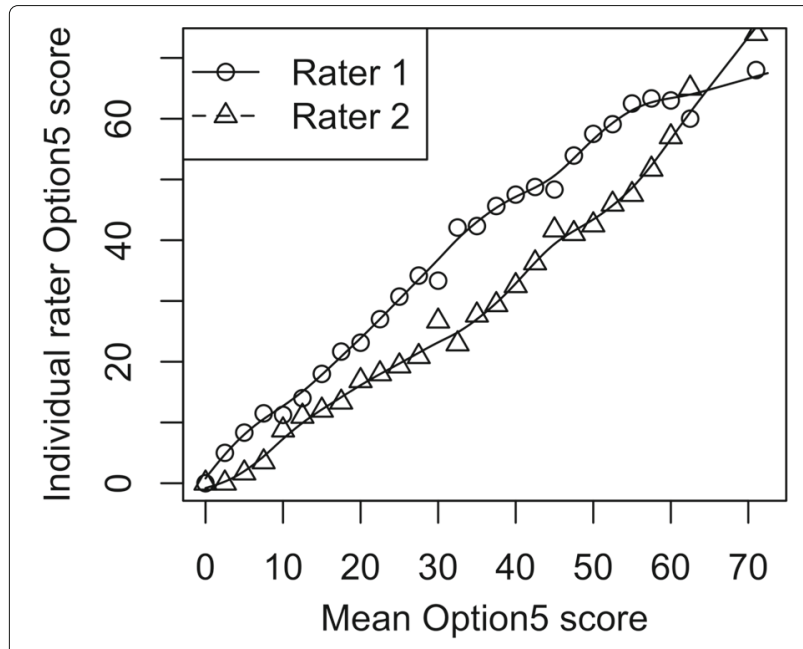

Fig. 1 Comparison of Observer OPTION ${ }^{5}$ scores between raters. The individual rater score is shown on the $y$-axis and the mean OPTION ${ }^{5}$ score is shown on the $x$-axis

variance is heteroscedastic and the sample mean variance across the encounters would yield a poor representation of the data. The binomial variance function performs better, suggesting that (6) may be an adequate model for the dependence of the variability of the raters' scores on the amount of SDM in the encounter.

A virtue of the Bayesian approach is that it avoids analytical approximations, even in complicated situations. The ICC is an example of a nonlinear function of parameters whose exact estimate and other inferences require variable transformation and high dimension integration to obtain the marginal posterior of the ICC. This happens

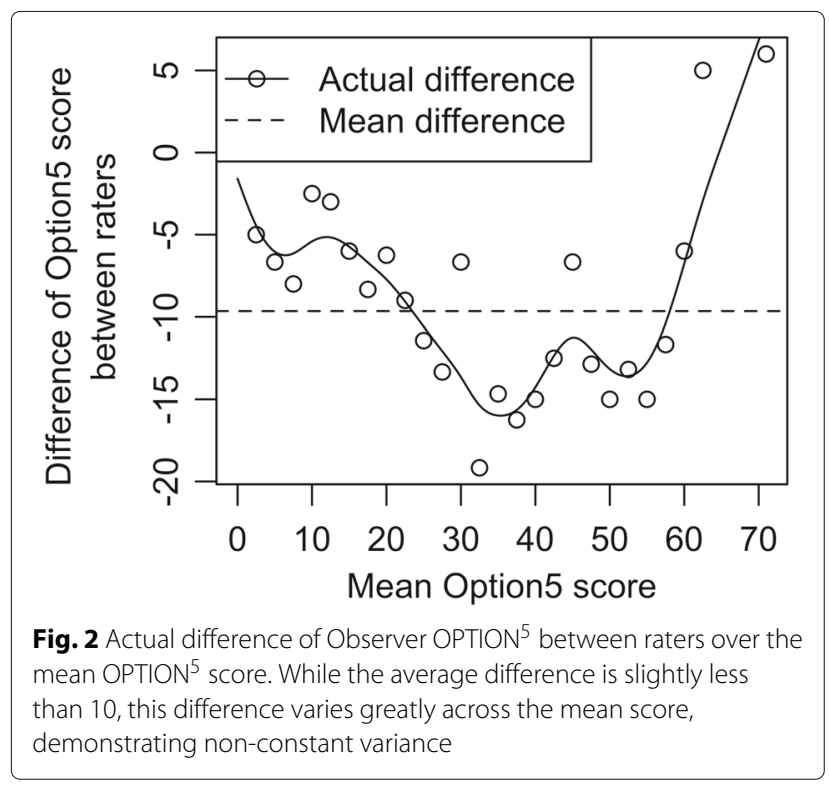

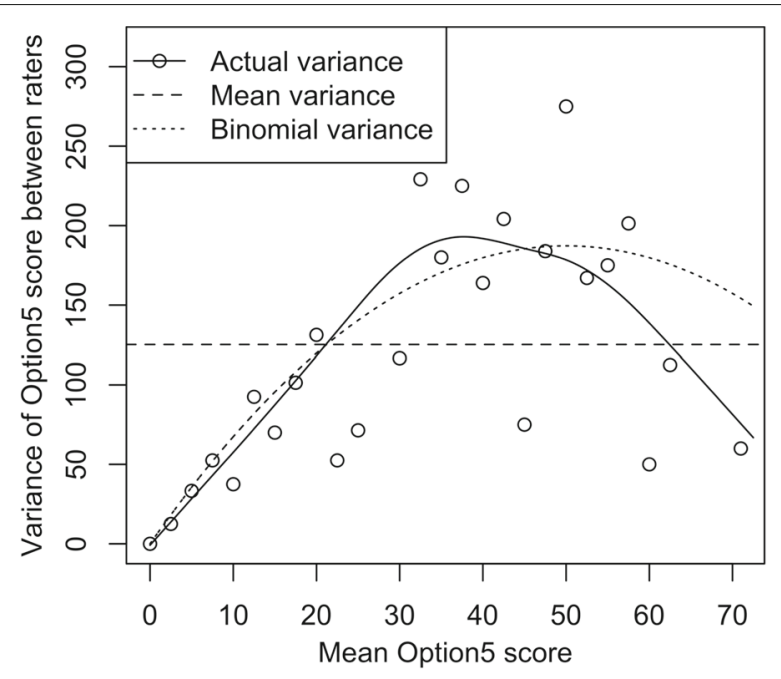

Fig. 3 Empirical variance of scores Compares the mean variance, binomial variance, and the observed variance (using a smoothing spline with 10 degrees of freedom) of Observer OPTION ${ }^{5}$ score. Highlights the heteroscedasticity of the variance as a function of the mean

automatically when Monte Carlo averages are evaluated over draws of parameters from the joint posterior distribution without requiring complicated mathematics to make accurate approximations. Hence, the joint posterior is used implicitly by the user.

To further illustrate the utility of our approach, we have produced plots of the posterior distribution of the key parameters underlying the ICC, the within-encounter variance and the between-encounter (within study) variance, for each study in Fig. 4. We also have made plots of the ICCs for each study and the difference in the ICC for each pair of studies in Fig. 5. In addition, we have also summarized the differences in the ICC between studies in terms of the posterior mean, posterior median, the 2.5 and 97.5 quantiles, and the posterior probability that the difference exceeds 0 in Table 2. Together, these figures and summary statistics provide a detailed description of the heterogeneity in the reliability of the measurement properties for each study and the statistical significance of differences between them. Such inferences are exact (to the numerical precision of the number of iterations we ran the MCMC procedure) and are trivial to obtain using Bayesian computation whereas more laborious and specialized calculations would be needed with frequentist calculation.

The hyperparameters for completing the specification of the Bayesian model are set to the values given above Eq. (8). Results from a full model, which takes the heterogeneity of the different studies into account are shown in Table 3. Summary ICC estimates for Study 1, 2, and 

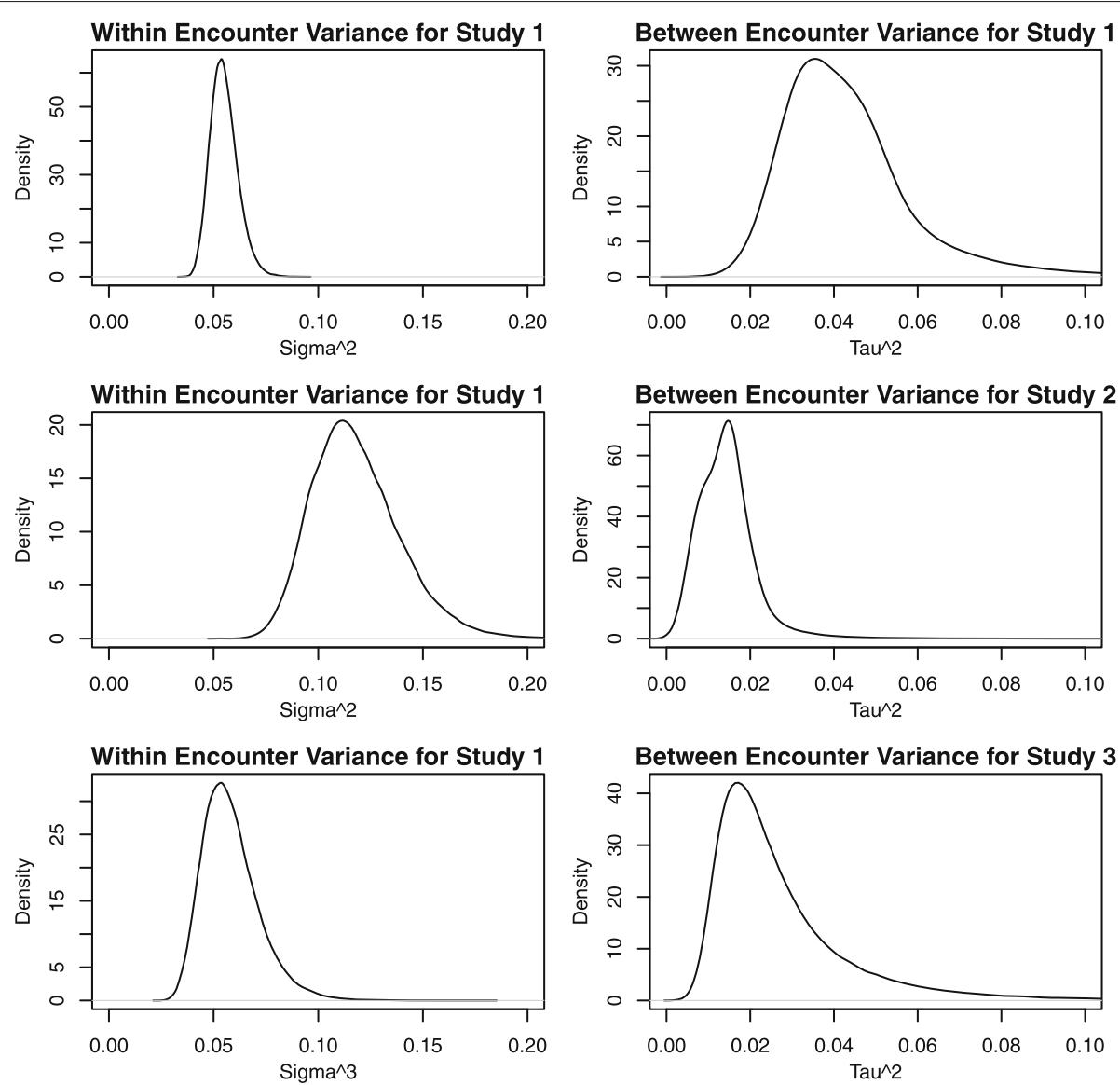

Fig. 4 A comparison of the posterior distribution of the key parameters underlying the ICC between the within-encounter variance and the between-encounter (but within study) variance across the three studies

3 were computed for a hypothetical new patient from the population of patients represented in each study (this corresponds to the population average ICC computed in Eq. 9). Note that the variance estimates are scaled to represent the rescaling of the OPTION ${ }^{5}$ score from $(0,100)$ to $(0,1)$. The resulting posterior means of the ICC were $0.821,0.295$, and 0.644 respectively. The estimate for Study 2 is particularly low in comparison to Study 1 (the posterior probability that study 1 has a higher ICC than study $2=0.995$ ) and Study 3 (the posterior probability that study 3 has a higher ICC than study $2=0.944$ ), demonstrating the extent of the heterogeneity between each study and how the ICC appraises very different impressions of the performance of Observer OPTION ${ }^{5}$ across the studies due to differences in the variability of SDM in the encounters it is trying to discriminate between. The credible intervals associated with the ICC estimates are quite wide due to the relative small sample sizes in two of the studies and the fact that there are only three studies to inform the between-study variance component, $\omega$.
The above results are further emphasized when we compare them to the results for the model with pooled study estimates and homogeneous variances using the same Bernoulli variance function for all three studies in Table 4. The pooled ICC estimate is 0.609 , a notable reduction for Study 1 and severely inflated compared to the separate estimate for Study 2. An interesting observation is that the estimate of the between study variance, $\omega$, is substantially less when study-specific estimates of the within- and between-encounter variances are not permitted (compare Table 4 to Table 3), illustrating how variation can be erroneously partitioned between levels of a model if there is substantial heterogeneity between the studies. We also tested a scenario for a pooled ICC estimate without accounting for the dependence of the within-encounter variance, hence assuming a constance variance across studies, on the true level of SDM in the encounter. We clearly see evidence of an inflated ICC with the estimated ICC of 0.640 (Table 5) substantially exceeding the pooled ICC estimate. If the study effects were ignored altogether (i.e., complete pooling of the data) then ICC estimates 

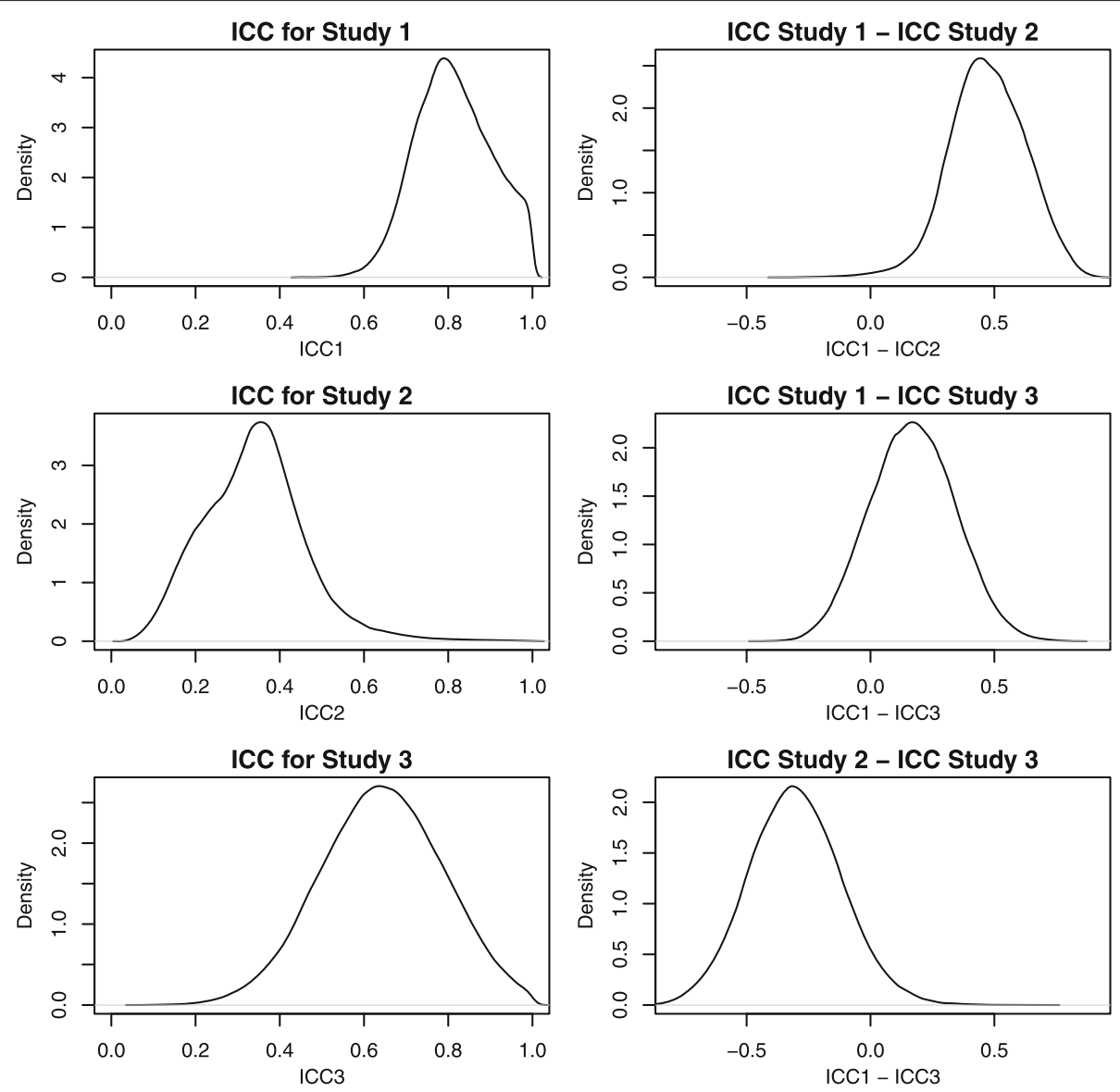

Fig. 5 Posterior distributions of the ICCS for each study, and the difference in the ICC for each pair of studies

were further inflated by approximately 0.02 (model not presented) while incorporating between study variation in the ICC via Eq. (10) yields estimates 0.072 or 0.066 greater depending on whether heteroscedasticity was accounted (Table 3) or ignored (Table 4).

Figure 6 illustrates the dependence of the ICC on the true amount of SDM in an encounter and the study in which the encountered occurred. The ICC trajectory lines for each study were constructed by evaluating the posterior mean of the ICC defined in (8) at 101 values of $\operatorname{SDM}\left(\theta^{*}\right)$ evenly spaced from 0 to 1 . Due to the concave shape of the variance function, encounters are easier to discriminate when the mean is closer to 0 or 100 than to 50 with the difference quite substantial. The contrasting

Table 2 The differences in the ICC between studies in terms of the posterior mean, median, the 2.5 and 97.5 quantiles, and the posterior probability that the difference exceeds 0

\begin{tabular}{llllll}
\hline Paired difference & $2.50 \%$ & Median & Mean & $97.50 \%$ & $p$-value \\
\hline Study 1 - Study 2 & 0.166 & 0.472 & 0.473 & 0.764 & 0.995 \\
Study 1 - Study 3 & -0.155 & 0.170 & 0.171 & 0.508 & 0.835 \\
Study 2 - Study 3 & -0.659 & -0.306 & -0.302 & 0.078 & 0.056 \\
\hline
\end{tabular}

Table 3 Full model results from Bayesian Framework*

\begin{tabular}{|c|c|c|c|}
\hline \multirow[t]{2}{*}{ Term } & \multicolumn{3}{|c|}{ Posterior summary } \\
\hline & Median & $2.5 \%$ & $97.5 \%$ \\
\hline$\beta[0]$ & 0.145 & -0.087 & 0.490 \\
\hline$\beta$ [Rater $]$ & -0.061 & -0.073 & -0.051 \\
\hline$\beta$ [Decision-aid] & 0.239 & 0.214 & 0.270 \\
\hline$(\sigma / 100)^{2}[$ Study 1$]$ & 0.054 & 0.044 & 0.070 \\
\hline$(\sigma / 100)^{2}[$ Study 2] & 0.117 & 0.084 & 0.168 \\
\hline$(\sigma / 100)^{2}[$ Study 3$]$ & 0.056 & 0.037 & 0.090 \\
\hline$\tau^{2}[$ Study 1] & 0.043 & 0.024 & 0.097 \\
\hline$\tau^{2}[$ Study 2] & 0.011 & 0.004 & 0.034 \\
\hline$\tau^{2}[$ Study 3$]$ & 0.023 & 0.009 & 0.078 \\
\hline$\omega$ & 0.029 & 0.003 & 0.717 \\
\hline ICC[Study 1] & 0.821 & 0.655 & 0.985 \\
\hline ICCStudy 2] & 0.295 & 0.119 & 0.628 \\
\hline ICC[Study 3] & 0.644 & 0.359 & 0.919 \\
\hline
\end{tabular}


Table 4 Results for homogeneous variance using a Bernoulli variance function to capture heteroscedastic variance

\begin{tabular}{|c|c|c|c|}
\hline \multirow[t]{2}{*}{ Term } & \multicolumn{3}{|c|}{ Posterior summary } \\
\hline & Median & $2.5 \%$ & $97.5 \%$ \\
\hline$\beta[0]$ & 0.317 & 0.203 & 0.449 \\
\hline$\beta$ [Rater $]$ & -0.088 & -0.102 & -0.074 \\
\hline$\beta$ [Decision-aid] & 0.250 & 0.217 & 0.281 \\
\hline$(\sigma / 100)^{2}$ & 0.041 & 0.035 & 0.048 \\
\hline$\tau$ & 0.015 & 0.011 & 0.019 \\
\hline$\omega$ & 0.004 & 0.001 & 0.092 \\
\hline ICC & 0.609 & 0.520 & 0.745 \\
\hline $\mathrm{ICCb} *$ & 0.681 & 0.568 & 0.935 \\
\hline
\end{tabular}

"ICCb denotes the ICC for the case when encounters are pooled across studies (see Eq. 10) whereas ICC is the conventional within-study ICC (see 8). In both cases the ICC is averaged over a population of encounters, as in 9

level of ICC across the three studies further emphasizes their heterogeneity.

\section{Discussion}

According to the COSMIN checklists, assessing the interrater reliability of instruments is essential when proposing novel health measurement scales $[9,10]$. These guidelines recommend assessing the ICC to examine the reliability of proposed measures, but only briefly allude to the limitations of broadly applying ICC (or reliability) estimates. As a result, the mass use of the ICC in the field of health measurement has lead to a variety of studies which may have miss-stated the reliability of new instruments from ignoring heteroscedasticity. For instance, Scholl et al review a collection of instruments with restricted scales that calculate ICC or inter-rater reliability many of whom do not account for the dependence of the variability of measurements on the value of the quantity being measured [28].

Table 5 Results for homogeneous variance using a constant variance function

\begin{tabular}{llll}
\hline \multirow{2}{*}{ Term } & \multicolumn{3}{l}{ Posterior summary } \\
\cline { 2 - 4 } & Median & $2.5 \%$ & $97.5 \%$ \\
\hline$\beta[0]$ & 0.319 & 0.206 & 0.451 \\
$\beta[$ Rater $]$ & -0.097 & -0.111 & -0.083 \\
$\beta[$ Decision-aid] & 0.278 & 0.248 & 0.309 \\
$(\sigma / 100)^{2}$ & 0.008 & 0.007 & 0.009 \\
$\tau$ & 0.014 & 0.011 & 0.017 \\
$\omega$ & 0.004 & 0.001 & 0.091 \\
ICC & 0.640 & 0.568 & 0.702 \\
ICCb* & 0.706 & 0.614 & 0.930 \\
\hline
\end{tabular}

"ICCb denotes the ICC for the case when encounters are pooled across studies (see Eq. 10) whereas ICC is the conventional within-study ICC (see 8). In both cases the ICC is averaged over a population of encounters, as in 9

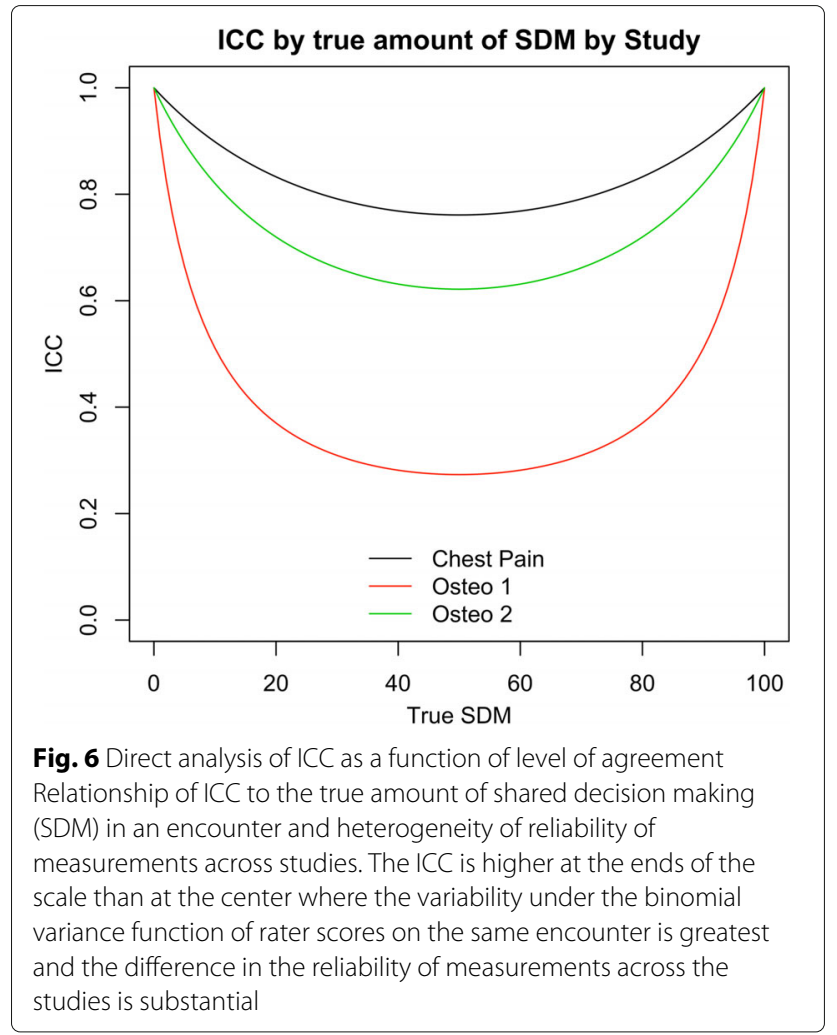

As well, there are many other studies of instruments with restricted scales which have given no indication that the assumption of homogenous variance has been met [39-41]. All of these studies are candidates for having miss-stated the ICC, and would benefit from implementing the framework proposed here. Furthermore, ICC estimates often have great implications in experimental design considerations, particularly in terms of properly powering studies. Hence, it is extremely important to have estimates that account for heteroscedasticity and apply to the context in which the instrument is planned to be used [22].

In a published guideline for selecting and reporting ICC measures, Koo and Li discuss 10 different forms of ICCs based on the model, type of measurement whether it be the mean of k-raters or a single-raters measurement, and whether or not absolute agreement or consistency is desired [14]. The measurement we've proposed here is an inter-rater, inter-case discriminatory ICC and hence applies for forms of the ICC considering multiple raters and emphasizing consistency of measurements.

We demonstrated that the ICC is inflated under a homoscedastic variance assumption and that multiple studies should not be pooled in order to calculate an overall ICC for an instrument when there is substantial heterogeneity between studies. We've proposed a framework which is robust to heteroscedastic variance while maintaining ease of interpretation for use by clinicians 
and other non-statisticians. As well, the implementation of a Bayesian framework negates the issue of a biased estimate for the ICC, as Bayesian estimates do not rely on closed-form approximations and normal distribution asymptotic theory [32, 42].

Because a wide variety of appraisals of Observer $\mathrm{OPTION}^{5}$ are possible using these data, it is possible that overly optimistic assessments could have been published and erroneously influenced research using Observer OPTION $^{5}$ to measure SDM (e.g., studies may be underpowered) if assumptions were not clearly described. We hope that the methodology outlined in this paper will be adopted widely and lead to correctly calibrated estimates and descriptions of ICC and, therefore, more informative profiles of instrument quality being used in important applications.

It should be noted, that while this approach will work for any case in which the measurement is bounded, it may be overkill when examining data from a single study where the outcome is tightly distributed around the middle of the scale. In that particular situation, conventional approaches for calculating the ICC should be satisfactory. In the absence of these conditions, the approach we are proposing here should be utilized.

An advantage of the framework developed in this paper is that it applies to any number of studies, raters, and encounters within studies. However, as the ICC is often calculated by non-statisticians, a potential limitation of this framework is the perceived learning curve in applying a Bayesian approach for estimation. Frequentist approaches dominate the medical literature, although it has been argued that clinicians naturally use Bayesian thinking in their everyday decision making [43]. To aid in the easy implementation of our framework, we deposited the $\mathrm{R}$ code used to generate our estimates in GitHub (see Availability of data and materials) along with the code we used to simulate data from the model described here to avoid potential patient data confidentiality issues.

While this paper mostly focused on the context of calculating an ICC in terms of developing measurement scales for health practitioners, this framework naturally extends into many other fields. Future work will include extending the approach to other fields of study.

\section{Conclusion}

Despite its wide-spread use as an important measure of inter-rater reliability, there are a variety of established methodological issues that need to be considered in specifying and estimating an ICC $[1,15-22]$. As it is a metric which is frequently applied by non-statisticians, there is concern that these issues are not properly being accounted for and, as a result, inflated ICC estimates are being published in the literature across a variety of fields. In this work, we propose a Bayesian framework for estimating the ICC that accounts for heteroscedastic variances and avoids relying on an estimator's normality for inferences to be correct. A particular strength of this approach is that it yields estimates which are robust to many common errors in ICC calculation while maintaining straightforward interpretation for researches across many fields of interest. Widespread adoption of this model-based ICC definition and allied estimation procedure would ultimately lead to more flexible and accurate representation of inter-rater reliability.

\section{Abbreviations}

COSMIN: Consensus-based standards for the selection of health status measurement instruments; ICC: Intraclass correlation coefficient; JAGS: Just another Gibbs sampler; MCMC: Markov chain Monte-Carlo; PDA: Personal decision aid; SDM: Shared decision making

\section{Acknowledgements}

The author's acknowledge the support of the Knowledge and Evaluation Research (KER) Unit at the Mayo Clinic (Rochester, MN, USA) in supplying the data used for the illustrative analyses.

\section{Funding}

No external support was received for this work. Dartmouth College holds an Institutional Program Unifying Population and Laboratory Based Sciences award from the Burroughs Wellcome Fund, and C. Bobak was supported by this grant (Grant\#1014106)

\section{Availability of data and materials}

A generated dataset that is structurally equivalent to the actual data as well as all code used to generate these analyses is available in the Bayesian Framework for InterRater ICC repository, https://github.com/CarlyBobak/ Bayesian-Framework-for-InterRater-ICC. The data that support the findings of this study are available from Victor M. Montori, M.D. upon reasonable request.

\section{Authors' contributions}

CB contributed to the drafting of the manuscript and the implementation of the framework in R and JAGS. PJB contributed to the study design of the illustrative application and drafting of the manuscript. AJO contributed to the design of the framework, original implementation in $\mathrm{R}$, and the drafting of the manuscript. All authors read and approved the final manuscript.

\section{Ethics approval and consent to participate}

N/A

\section{Consent for publication}

N/A

\section{Competing interests}

The authors declare that they have no competing interests.

\section{Publisher's Note}

Springer Nature remains neutral with regard to jurisdictional claims in published maps and institutional affiliations.

Received: 23 November 2017 Accepted: 23 August 2018

Published online: 12 September 2018

\section{References}

1. Fisher RA. On the "probable error" of a coefficient of correlation deduced from a small sample. Metron. 1921;1:1-32.

2. Ebel RL. Estimation of the reliability of ratings. Psychometrika. 1951;16: 407-24. https://doi.org/10.1007/bf02288803

3. Strah KM, Love SM. The in situ carcinomas of the breast. J Am Med Women's Assoc (1972). 1992;47:165-8.

4. Visscher PM, Medland SE, Ferreira MAR, Morley Kl, Zhu G, Cornes BK, Montgomery GW, Martin NG. Assumption-free estimation of heritability 
from genome-wide identity-by-descent sharing between full siblings. PLoS Genet. 2006;2:41. https://doi.org/10.1371/journal.pgen.0020041.

5. Bradley RA, Schumann DEW. The comparison of the sensitivities of similar experiments: Applications. Int Biom Soc. 1957;13:496. https://doi.org/10. 2307/2527974.

6. Pellis $L$, Hal NLWF-V, Burema J, Keijer J. The intraclass correlation coefficient applied for evaluation of data correction, labeling methods, and rectal biopsy sampling in dna microarray experiments. Physiol Genomics. 2003;16:99-106. https://doi.org/10.1152/physiolgenomics. 00111.2003.

7. Killip S. What is an intracluster correlation coefficient? crucial concepts for primary care researchers. Ann Fam Med. 2004;2:204-8. https://doi.org/10. 1370/afm.141.

8. Ukoumunne OC, Davison AC, Gulliford MC, Chinn S. Non-parametric bootstrap confidence intervals for the intraclass correlation coefficient. Stat Med. 2003;22:3805-21. https://doi.org/10.1002/sim.1643.

9. Mokkink LB, Terwee CB, Patrick DL, Alonso J, Stratford PW, Knol DL, Bouter LM, de Vet HC. COSMIN checklist manual. Amst Univ Med Cent. 2012.

10. Mokkink LB, Terwee CB, Patrick DL, Alonso J, Stratford PW, Knol DL, Bouter LM, de Vet HCW. The COSMIN checklist for assessing the methodological quality of studies on measurement properties of health status measurement instruments: an international delphi study. Qual Life Res. 2010;19:539-49. https://doi.org/10.1007/s11136-010-9606-8.

11. McGraw KO, Wong SP. Forming inferences about some intraclass correlation coefficients. Psychol Methods. 1996;1 (1):30-46. https://doi. org/10.1037/1082-989x.1.1.30.

12. Fisher RA. Statistical methods for research workers. In: Breakthroughs in statistics. Springer; 1992. p. 66-70.

13. Shrout PE, Fleiss JL. Intraclass correlations: uses in assessing rater reliability. Psychol Bull. 1979;86(2):420.

14. Koo TK, Li MY. A guideline of selecting and reporting intraclass correlation coefficients for reliability research. J Chiropr Med. 2016;15(2): 155-63. https://doi.org/10.1016/j.jcm.2016.02.012.

15. Altaye $\mathrm{M}$, Dormer $\mathrm{A}$, Klar N. Inference procedures for assessing interobserver agreement among multiple raters. Biometrics. 2001;57(2): 584-8.

16. Ghosh H, Das A. Optimal diallel cross designs for estimation of heritability. Stat Med. 2003;116:185-96. https://doi.org/10.1016/s03783758(02)00180-5.

17. Shoukri M, Donner A. Efficiency considerations in the analysis of inter-observer agreement. Biostatistics. 2001;2(3):323-36.

18. Konishi S. Normalizing and variance stabilizing transformations for intraclass correlations. Ann Inst Stat Math. 1985;37(1):87-94.

19. Weinberg R, Patel YC. Simulated intraclass correlation coefficients and their z transforms. J Stat Comput Simul. 1981;13(1):13-26.

20. Wang CS, Yandell BS, Rutledge JJ. Bias of maximum likelihood estimator of intraclass correlation. Theor Appl Genet. 1991;82:421-4. https://doi. org/10.1007/bf00588594.

21. Ponzoni R, James J. Possible biases in heritability estimates from intraclass correlation. Theor Appl Genet. 1978:53(1):25-7.

22. Atenafu EG, Hamid JS, To T, Willan AR, Feldman BM, Beyene J, Bias-corrected estimator for intraclass correlation coefficient in the balanced one-way random effects model. BMC Med Res Methodol. 2012;12.https://doi.org/10.1186/1471-2288-12-126.

23. Olkin I, Pratt JW. Unbiased estimation of certain correlation coefficients, Ann Math Stat. 1958;29:201-11. https://doi.org/10.1214/aoms/ 1177706717.

24. Shay LA, Lafata JE. Where is the evidence? a systematic review of shared decision making and patient outcomes. Med Dec Making. 2014;35(1): 114-31. https://doi.org/10.1177/0272989x14551638.

25. Elwyn G, Frosch D, Thomson R, Joseph-Williams N, Lloyd A, Kinnersley P, Cording E, Tomson D, Dodd C, Rollnick S, Edwards A, Barry M. Shared decision making: A model for clinical practice. J Gen Intern Med. 2012;27(10):1361-7. https://doi.org/10.1007/s11606-012-2077-6.

26. Barr PJ, Elwyn G. Measurement challenges in shared decision making: putting the 'patient' in patient-reported measures. Health Expect. 2015;19(5):993-1001. https://doi.org/10.1111/hex.12380.

27. Fitzpatrick R. Surveys of patients satisfaction: I-important general considerations. Br Med J. 1991;302(6781):887.
28. Scholl I, Loon MK-v, Sepucha K, Elwyn G, Légaré F, Härter M, Dirmaier J. Zeitschrift für Evidenz, Fortbildung und Qualität im Gesundheitswesen. 2011;105(4):313-24. https://doi.org/10.1016/j.zefq.2011.04.012.

29. Dillon EC, Stults CD, Wilson C, Chuang J, Meehan A, Li M, Elwyn G, Frosch DL, Yu E, Tai-Seale M. An evaluation of two interventions to enhance patient-physician communication using the observer OPTION ${ }^{5}$ measure of shared decision making. Patient Educ Couns. 2017;100(10): 1910-7.

30. Barr PJ, O'Malley AJ, Tsulukidze M, Gionfriddo MR, Montori V, Elwyn G. The psychometric properties of observer OPTION5, an observer measure of shared decision making. Patient Educ Couns. 2015;98(8):970-6. https:// doi.org/10.1016/j.pec.2015.04.010

31. Elwyn G, Lloyd A, May C, van der Weijden T, Stiggelbout A, Edwards A, Frosch DL, Rapley T, Barr P, Walsh T, Grande SW, Montori V, Epstein R. Collaborative deliberation: A model for patient care. Patient Educ Couns. 2014;97(2):158-64. https://doi.org/10.1016/j.pec.2014.07.027.

32. Bartlett JW, Keogh RH. Bayesian correction for covariate measurement error: A frequentist evaluation and comparison with regression calibration. Stat Methods Med Res. 2014;0(0):0962280216667764. https:// doi.org/10.1177/0962280216667764.

33. Gelman A. Prior distributions for variance parameters in hierarchical models. Bayesian Anal. 2006;1:1-19.

34. R Core Team. R: A Language and Environment for Statistical Computing. Vienna, Austria: R Foundation for Statistical Computing; 2016. https:// www.R-project.org/.

35. Plummer M, et al. JAGS: A program for analysis of Bayesian graphical models using Gibbs sampling. In: Proceedings of the 3rd international workshop on distributed statistical computing, Vol. 124 no. 125.10. Vienna; 2003

36. Plummer M. Rjags: Bayesian Graphical Models Using MCMC. 2016. R package version 4-6. https://CRAN.R-project.org/package=rjags.

37. Hess EP, Knoedler MA, Shah ND, Kline JA, Breslin M, Branda ME, Pencille LJ, Asplin BR, Nestler DM, Sadosty AT, Stiell IG, Ting HH, Montori VM. The chest pain choice decision aid: A randomized trial. Circ Cardiovasc Qual Outcomes. 2012;5(3):251-9. https://doi.org/10.1161/ circoutcomes.111.964791.

38. Montori VM, Shah ND, Pencille LJ, Branda ME, Houten HKV, Swiglo BA, Kesman RL, Tulledge-Scheitel SM, Jaeger TM, Johnson RE, Bartel GA, Melton $L$, Wermers RA. Use of a decision aid to improve treatment decisions in osteoporosis: The osteoporosis choice randomized trial. Am J Med. 2011;124(6):549-56. https://doi.org/10.1016/j.amjmed.2011.01.013.

39. Revicki DA, Leidy NK, Brennan-Diemer F, Sorensen S, Togias A. Integrating patient preferences into health outcomes assessment. Chest. 1998;114(4):998-1007. https://doi.org/10.1378/chest.114.4.998.

40. Fried TR, Bradley EH, Towle VR. J Gerontol Ser B Psychol Sci Soc Sci. 2002;57(6):348-54.

41. Munters LA, van Vollenhoven RF, Alexanderson H. Patient preference assessment reveals disease aspects not covered by recommended outcomes in polymyositis and dermatomyositis. ISRN Rheumatol. 2011;2011:1-5. https://doi.org/10.5402/2011/463124.

42. Giere RN. Bayesian statistics and biased procedures. Synthese. 1969;20(3): 371-87.

43. Gill CJ. Why clinicians are natural bayesians. BMJ. 2005;330(7499):1080-3. https://doi.org/10.1136/bmj.330.7499.1080. 